\title{
Sistema de Formación Pedagógica en la Universidad de Otavalo en Ecuador
}

\author{
Luis A. Acosta, Omar Abreu y Marcela F. Coronel \\ Universidad de Otavalo, Campus: Cdla Imbaya. Av. de los Sarances $s / n$ y Pendoneros, Otavalo, Ecuador \\ (e-mail: lacosta@uotavalo.edu.ec, oabreu@uotavalo.edu.ec, mcoronel@uotavalo.edu.ec)
}

Recibido Sep. 3, 2014; Aceptado Oct. 23, 2014; Versión final recibida Nov. 6, 2014

\begin{abstract}
Resumen
El objetivo de este artículo es analizar el sistema de perfeccionamiento en formación pedagógica de los profesores del claustro de la Universidad de Otavalo en Ecuador. Se aplica una concepción sustentada en la teoría y la práctica para la preparación del claustro desde el punto de vista pedagógico, considerando el contexto, la definición y la caracterización de los niveles de formación de los profesores para perfeccionar la dimensión pedagógica: formativo, básico superior, actualización y profundización. Los resultados muestran que la aplicación del sistema de formación pedagógica contribuye a la elevación de la competencia profesional del claustro de profesores. Esto influye en la captación de mejores estudiantes y en una más alta retención de los estudiantes en la Universidad.
\end{abstract}

Palabras claves: perfeccionamiento continuo, formación pedagógica, destrezas docentes, retención de estudiantes

\section{Pedagogical Formation System of the Otavalo University in Ecuador}

\begin{abstract}
The aim of this article is to analyze the training on pedagogical skills of professors of the University of Otavalo in Ecuador. Theoretical and practical concepts to prepare faculty from the pedagogical point of view are applied. The pedagogical training system takes into account the context, the definition and the characterization of the teachers' pedagogical level: basic, intermediate, updating stage and deepening stage. The results show that the application of the present pedagogical training system increases the teaching professional skills of professors. This has a positive effect on the recruitment of better students and help in maintaining a higher retention of student in the University.
\end{abstract}

Keywords: continuing education, pedagogical formation, teaching skills, student retention 


\section{INTRODUCCIÓN}

El mundo moderno ha impuesto nuevos retos en todas sus manifestaciones. Uno de los más rigurosos se relaciona con el saber, con el aprendizaje, el dominio y la creación del conocimiento, lo que ha concentrado la atención en el desarrollo educativo y particularmente en las universidades. "Con el advenimiento de la globalización se anuncia una nueva era, la del conocimiento o sociedad de los saberes (...) la educación pasa a ser considerada el centro de la agenda disponible para que los Estados (nacionales 0 transnacionales) enfrenten esa nueva estructuración del mundo. La Educación Superior representa la base de la continuidad del proceso de desarrollo económico y justicia social (...)". (Lucchesi, 2011)

El número de centros educativos interesados en elevar el rigor en el desempeño profesional de sus claustros docentes, crece sistemáticamente en la medida en que sus directivos toman conciencia en relación con la necesidad de tener docentes competentes, para cumplir exitosamente encargos sociales cada vez más exigentes. "Actualmente existen algunas instituciones educativas que empiezan a tomar conciencia sobre la necesidad de crear nuevas políticas y estrategias orientadas a reducir la brecha entre el currículum, la investigación y la práctica pedagógica (...)" (Armas y Fernández 2009).

Los centros educacionales no pueden cumplir con la responsabilidad que determinó sus orígenes, si los claustros que los integran no poseen una preparación de carácter pedagógico bien concebida y profunda. "La sociedad fomenta y desarrolla un conjunto de instituciones educativas cuya misión fundamental es educar de manera organizada, sistemática y coherente a las nuevas generaciones, en estas instituciones el proceso educativo se desarrolla sobre la base de una determinada concepción pedagógica, es conducida por personal con formación pedagógica y disponen de los medios y recursos imprescindibles para cumplir el encargo social de manera más eficiente". (Pla, et al., 2010).

La demanda de un ser humano con una formación integral y contextualizada, a la altura de los niveles de desarrollo de la diversidad contemporánea, reclama una revolución continua en la formación de profesores universitarios, responsables de tan complejo proceso. "En el contexto de la sociedad del conocimiento, las universidades han cobrado un importante rol en la formación de capital humano avanzado (...) en este caso de la formación docente". (Pedraja, et al., 2012). El esfuerzo y la consagración de los profesores universitarios a una preparación y superación continuas, es la manera más efectiva de responder a los requerimientos históricos vigentes, porque "Los tiempos que corren están exigiendo de quienes se dedican a la formación de profesionales un estado de permanente alerta, de reconceptualización e indagación de nuevas y viejas herramientas, a la luz de las demandas sociales de aprender de otra manera." (Alvariñas y Fernández, 2011).

La formación pedagógica del profesorado universitario es uno de los grandes desafíos de la educación, y constituye la base para la solución de otros problemas planteados a esta. García (1996) considera que el autoperfecionamiento docente "es la actividad autotransformadora que presupone el cambio del docente centrado en el dominio y comprensión profunda de los fines y naturaleza de la actuación profesional, incluyendo los mecanismos que facilitan su cambio sistemático y su autoanálisis". Así mismo continúa insistiendo García (1996) en que "autoperfeccionarse es un constante reanálisis de la información sobre los modos de actuar, los procedimientos, las motivaciones, las conceptualizaciones sobre la labor pedagógica, que generan procesos de búsqueda y transformaciones a partir de la propia experiencia y de la experiencia ajena y que recodifica, reorganiza y sistematiza todo el sistema de trabajo, hacia estadios superiores de desarrollo conscientemente determinados". La actualidad del debate científico en relación con esta problemática es una referencia constante en la literatura especializada y en publicaciones de autores vinculados a ella. Para Abreu (2014) "La formación profesional de profesores (...) con un elevado nivel científico, con independencia cognoscitiva y firme compromiso patriótico, es una tarea de primer orden, necesidad impostergable y elevada complejidad para toda sociedad empeñada en subsistir y desarrollarse con la participación plena de todos sus integrantes".

Para el desarrollo exitoso de todo modelo educativo, es imprescindible el nivel de dominio sobre la teoría y la práctica pedagógicas que alcance el claustro pues "La categoría de mayor grado de esencialidad y generalidad que asume la Pedagogía como ciencia es la educación entendida en esta concepción como la preparación de la persona para aportar al desarrollo de la sociedad, que se expresa en una actitud transformadora y creadora en todos los contextos, se alcanza con el aprendizaje de los conocimientos, las habilidades, los valores, los métodos y modos de actuación acumulados en la cultura del hombre, a través de actividades, que pueden organizarse como un proceso de enseñanza sistemático, intencionalmente concebido en relación con los fines de la sociedad, con unos contenidos especialmente seleccionados, para desarrollar una dinámica comunicativa estructurada temporal y espacialmente, en relación con las características individuales y colectivas de los sujetos que intervienen y que requiere de una dirección pedagógica". (Ramos, et al., 2013). 
Armas y Fernández (2009) en "Formación Pedagógica Docente y Desempeño Académico de Alumnos en la Facultad de Ciencias Administrativas de la UABC", plantean que "La formación docente es aquella que posibilita el desarrollo de competencias propias del ejercicio profesional en los diferentes niveles y modalidades del sistema educativo". La complejidad, el dinamismo, la dialéctica y la necesidad de este proceso hacen de "La formación docente (...) un proceso permanente, dinámico, integrado, en el que convergen, entre otros elementos, la disciplina y sus aspectos teóricos, metodológicos, epistemológicos, didácticos, psicológicos, sociales, filosóficos e históricos, para lograr la profesionalización de la docencia". (Chehaybar y Amador, 2003).

Para las universidades resulta pertinente dotar a los docentes de las herramientas, vías o mecanismo que desarrollen la capacidad de autoanálisis para lograr una transformación consciente y el tránsito hacia posiciones más exigentes del desarrollo pedagógico. Se ha demostrado la persistencia de una baja actividad metacognitiva en los estudiantes, incluyendo los niveles superiores de la enseñanza, como reflejo de la manifestación de este problema en sus profesores. El buen profesor ha de proporcionar guías, modelos, herramientas para orientar la actividad mental de sus alumnos hacia el logro de objetivos de aprendizaje, hacia la apropiación del nuevo conocimiento. Este desempeño profesional es posible cuando el educador tiene una preparación pedagógica que facilita la utilización armónica y balanceada de los recursos didácticos oportunos, para estimular y conducir el aprendizaje de los estudiantes. (Rosenshine at al., 1996)

\section{FORMACIÓN DOCENTE}

Las tendencias educativas del nuevo siglo han impuesto una dinámica diferente y más rigurosa en relación con el modo de actuación profesional de los docentes. "La educación para el siglo XXI, exige educadores innovadores, creativos, investigativos e inquietos por los procesos de enseñanza aprendizaje, desde una realidad cambiante que genere estrategias y oportunidades para que los educandos asuman los desafíos de una manera responsable y autónoma (...). El perfil del educador hoy en día, apunta a una enseñanza orientada a la producción lógica de conocimientos, desde una participación activa del estudiante invitándolo a discutir sus ideas y a compartir sus saberes, dejando de lado la monotonía y los procesos mecánicos y repetitivos que encasillan al estudiante en un rol tradicional (...). Tarea no sencilla de lograr a partir del simple conocimiento que el docente posee sobre la disciplina objeto de enseñanza, sino a través de las actitudes y comportamientos asumidos por él, siendo un ente articulador de la ciencia, la tecnología y la educación desde una mirada problematizadora y contextualizada". (Moreno at al., 2014).

Armas y Fernández (2009) también establecen una clara relación dialéctica entre la formación docente y el efecto transformados en los estudiantes cuando afirman que "El resultado final persigue ofrecer alternativas que posibiliten una educación de mayor calidad, ya que como bien indica "(...) parce claro por diversas estudios al respecto (...) que los programas de formación, capacitación, actualización de profesores, tienen una incidencia en la calidad de los servicios educativos", lo cual implica que para elevar la calidad de la educación superior, es de suma importancia estructurar un vigoroso programa de formación de profesores". Refiriéndose concretamente al estudio que originó el artículo mencionado plantean ambas autoras, Armas y Fernández (2009), que "La relevancia de este estudio consiste en que los alumnos se verán beneficiados, en tanto esperamos obtener una educación con mayor calidad, lo cual a su vez, favorecería el nivel sociocultural de la sociedad en general".

El mismo vínculo plantea, con aguda visión pedagógica, Álvarez (2010) cuando coloca sobre la mesa de análisis realidades comunes del desarrollo de procesos concernientes a la educación y demuestra que "en el centro de dichos procesos se encuentra la metodología de enseñar y la de aprender, haciendo énfasis en esta última, como parte de una concepción pedagógica y didáctica que pone en el corazón del proceso educativo al alumno" y que establece nuevos compromisos de superación permanente, para los profesionales de este decisivo sector de la sociedad.

Resulta útil insistir en el significado de la formación de docentes universitarios para perfeccionar su modo de actuación profesional, en la necesidad de estimular la dedicación y la entrega a la profesión, en la autopreparación sistemática y consciente, como un rasgo distintivo de los profesores de la Educación Superior, para desarrollar los conocimientos, las habilidades y los valores y en la preparación armónica e integral que se demanda en estos profesores, cuyo medio ideal es el postgrado. La formación del docente ha sido vista desde diferentes paradigmas, Pedagogía Tradicional, Pedagogía Crítica, Pedagogía Liberadora y Pedagogía Constructivista, como parte de la formación permanente, asociada a las exigencias pedagógicas y a sus competencias inherentes. Varios autores fundamentan sus posiciones en sistemas, modelos y programas que se ofertan en instituciones de Educación Superior. Algunos 
profundizan en el carácter cada vez más humanista y menos tecnológico que debe tener el proceso de formación sustentado pedagógicamente y que necesita el profesor universitario.

Al analizar los componentes del proceso de formación de los docentes, Álvarez (1999) expresa que "el componente académico: contiene el fundamento teórico necesario de los objetivos del programa y básicamente está contenido en las asignaturas, disciplinas o módulos, y carreras donde se desempeña el docente. El componente laboral: abarca toda la actividad práctica a realizar, concebida como un sistema enfocado hacia alcanzar el modo de actuación requerido. Está íntimamente relacionada con su actividad diaria como profesor., es decir, potenciar el acercamiento de la academia a lo laboral e investigativo. Tributa con mucha fuerza a la profesionalización del profesor universitario. El componente investigativo: abarca la actividad del profesor como constructor de los resultados de la investigación realizada, es decir, el profesor capacitado para aplicar los adelantos de la ciencia de la educación".

Es necesario articular la teoría con la práctica, como condición para el desarrollo de investigaciones que respondan a la solución de necesidades propias de esta ciencia, la Pedagogía, y que sean sostenibles, luego que el profesor transite de formas más simples a las más complejas en su formación, atendiendo a las necesidades de su nivel de desarrollo profesional. En Ecuador la formación del docente universitario se asume a corto y mediano plazos y contextualizada en cursos, conferencias, seminarios y talleres. Es un punto de partida imprescindible en estas instituciones ecuatorianas y en particular en la Universidad de Otavalo. Se ha considerado el diagnóstico de necesidades de los profesores en la dimensión pedagógica, como el cimiento para la ubicación del claustro por niveles de desarrollo alcanzado en esta área del conocimiento y como el centro de dicho proceso. (Addine, et al., 2004)

En la aplicación de esta alternativa de formación continua se logró, la preparación para un ejercicio responsable, ético y pertinente de la docencia, expresado en la autonomía y el compromiso con la calidad de la función educativa, que las vías utilizadas para alcanzar tal fin se diseñen, ejecuten y evalúen y se expresen en indicadores relacionados con el desarrollo el modo de actuación profesional como: interés por lo que se hace, satisfacción en el desempeño docente, reflexión crítica acerca del quehacer como profesor, perseverancia, compromiso con la aspiración de alcanzar la calidad educativa, flexibilidad en la labor educativa y dominio de conocimientos y habilidades profesionales. Es una demanda de primer orden que la formación docente responda a las necesidades del profesorado universitario, teniendo en consideración la diversidad de contextos en que actúa y que esta tenga un carácter permanente, flexible y diferenciado por niveles de desarrollo, para relacionar los intereses individuales e institucionales. El desarrollo del hombre como individuo, se asocia a la pedagogía, cuando se emplea como herramienta del docente, para interactuar sobre él con acciones diferenciadas y para ejercer su actividad y los procesos de institucionalización universitaria.

El conocimiento de los fenómenos educacionales, desempeña una función importante en el enfoque de sistema que proporciona la orientación general para su estudio, como una realidad integrada por componentes que cumplen determinadas funciones y mantienen formas estables de interacción entre ellos. Un sistema no es un conglomerado de elementos yuxtapuestos mecánicamente. Representa leyes de totalidad; cualidades generales inherentes al conjunto, las cuales se diferencian de las características individuales de los componentes que lo integran y exige una dinámica dialéctica entre todas sus partes. Los fenómenos educacionales, están sujetos a leyes que los caracterizan como sistema y estudiarlos es muy valioso para el dominio de la metodología de la investigación pedagógica. Las cualidades en cuestión son: componentes, principio de jerarquía, estructura, y relaciones funcionales del sistema.

\section{CLAUSTRO DE LA UNIVERSIDAD DE OTAVALO}

El sistema propuesto para la formación del claustro de la universidad de Otavalo, comprende acciones conscientes, tiene carácter continuo, sistémico, personalizado, contextualizado y evaluable. Su propósito es lograr la preparación pedagógica, para resolver situaciones docentes en las aulas universitarias contemporáneas, para responder a las exigencias y expectativas de la educación del siglo XXI. Los estudios teóricos y prácticos posibilitaron la elaboración de un sistema de formación con enfoque pedagógico cuyos rasgos distintivos se revelan en la determinación de niveles, componentes y en las relaciones entre ellos, atendiendo a la necesidad de una estructura orgánica, revelada en la unidad de sus propiedades, componentes o partes constituyentes. Su diseño se fundamenta en la concepción teórica de la formación centrada en las carencias pedagógicas del claustro, que exige niveles para su realización. Contienen los elementos que fundamentan la preparación de los profesores, teniendo en cuenta el objetivo, el objeto y el eje transversal del proceso y la realización del diagnóstico inicial. Conjuga las necesidades reveladas, con los intereses profesionales, lo que originó un sistema de formación docente, perfeccionado, fortalecido y desarrollador de conocimientos, habilidades, valores y de un modo de actuación del profesor universitario, en correspondencia con las demandas sociales planteadas a la Universidad de Otavalo. En la 
concepción y aplicación del sistema de formación de los profesores se practicó la evaluación-mejora, como elemento permanente para perfeccionar el desempeño del profesor. El sistema se aplicó de forma escalonada, y su ejecución, desarrollo e implementación fue programada para los años del 2010 al 2014. Esquemáticamente, se representó como se observa en la figura 1.

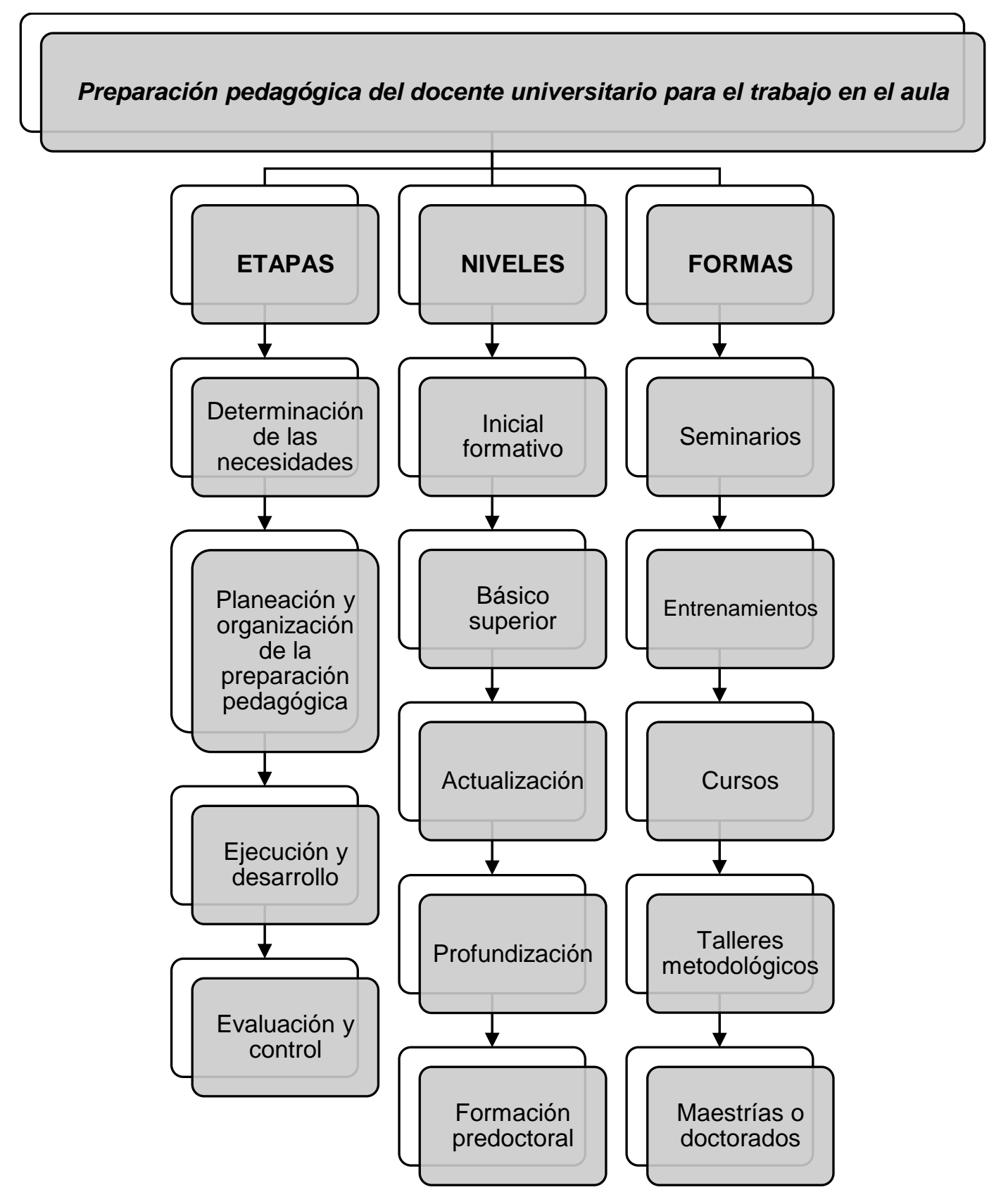

Fig. 1: Esquema del sistema de formación pedagógica del claustro en la Universidad de Otavalo

Es apreciable la existencia de relaciones de interdependencia e interacción entre los componentes del sistema, entre las etapas, los niveles y las formas de implementación. Resalta la coordinación entre los tres componentes y es evidente que los niveles son componentes centrales, a los que se subordinan los restantes. Los niveles de formación son los diferentes estadios de desarrollo de las habilidades docentes del claustro, desde el básico hasta el predoctoral, que proporcionan toda la superación, de carácter básico y generalista y de mayor extensión y profundización en la teoría del conocimiento y la práctica pedagógica con carácter aplicado y creativo. Se determinaron a partir de un basamento científico, tecnológico, social y procedimental con el propósito de garantizar que los profesores estén habilitados para un desempeño profesional solvente para enfrentar con éxito contingencia profesionales. Los niveles de formación propuestos son nivel inicial formativo, nivel básico superior, nivel de actualización, nivel de profundización y nivel de formación predoctoral. 
Nivel inicial formativo. Incluye a todos los estudiantes que se encuentren cursando las carreras que se estudian en la Universidad de Otavalo. Recibirán el Programa de la materia Pedagogía como asignatura obligatoria de 32 horas en el currículo. Persigue desarrollar las capacidades, disposiciones y actitudes de los profesores preparándolos para acceder a la enseñanza y facilitarles la eficacia en su tarea docente. Los resultados esperados en este nivel de formación pedagógica son: i) Formación de valores morales, éticos y profesionales en los estudiantes, priorizando la honestidad y la solidaridad de gran importancia en la labor pedagógica; ii) Desarrollo del pensamiento científico e investigativo que debe caracterizar a un profesional de las ciencias técnicas y humanísticas, promoviendo el espíritu investigativo, la superación y la creatividad en su actividad laboral; y iii) Desarrollo del liderazgo y la capacidad de trabajo en grupo.

Nivel básico superior. Incluye a aquellos docentes y directivos con tercer nivel vencido en ramas del conocimiento diferentes a las Ciencias Pedagógicas, que no acreditan formación pedagógica, pero poseen años de experiencia en la Educación Superior u otro nivel de enseñanza. Está relacionado con la fase de inducción expresada en la literatura especializada y se corresponde en el tiempo con los primeros años de ejercicio, en los que los docentes universitarios aprenden en la práctica. Incluye tres tipos de situaciones profesionales: profesores noveles que terminado sus estudios acceden por primera vez a la enseñanza universitaria como profesionales; profesores que regresan a la enseñanza después de varios años de distanciamiento del desempeño como docente y profesores que desempeñan nuevas tareas y necesitan de una formación específica. Los resultados en este nivel de formación pedagógica son : i) Perfeccionamiento de la labor docente-educativa de los profesores mediante una formación en los aspectos pedagógicos fundamentales que caracterizan el proceso enseñanza-aprendizaje en la Educación Superior y en especial en el Ecuador; y ii) Formación de los profesores universitarios, en los aspectos que caracterizan el proceso docente-educativo en la Educación Superior, para que apliquen los conocimientos, habilidades, valores y modos de actuación, adquiridos en los salones de las clases mediante reflexiones teóricas y prácticas.

Nivel de actualización. Incluye a aquellos docentes y directivos con tercer nivel vencido en Ciencias Pedagógicas, que no acreditan cursos de actualización pedagógica en los últimos cinco años. Se relaciona con algunas etapas descritas en la literatura especializada. Se reconoce como un momento o etapa de formación en ejercicio, continua, de perfeccionamiento, actualización, reciclaje, desarrollo profesional y formación en servicio. Su principal característica es que incluye aquellas actividades que propician el desarrollo profesional del docente. Los resultados en este nivel de formación pedagógica son: i) - Contribuir a la formación permanente y a la profundización de los participantes en diferentes campos de la administración educativa enfocada a la dirección científica del proceso docente-educativo que se desarrolla en las instituciones de educación superior; ii) Analizar con enfoque de sistema el proceso docente educativo y las regularidades que se manifiestan en éste, valorando las funciones de planificación, organización, integración, dirección y control; y iii) Promover el estudio comparado de estas temáticas y dar a cada participante la oportunidad de evaluar aspectos importantes de la dirección del proceso docente-educativo, de especial interés para la Universidad de Otavalo.

Nivel de profundización. Incluye a aquellos docentes y directivos que acreditan cursos de actualización pedagógica dentro de los últimos cinco años. Estos deben integrarse directamente a los programas de maestría relacionados con las Ciencias Pedagógicas. Los resultados en este nivel de formación pedagógica son desarrollar capacidades en los profesores para la gestión y la innovación en instituciones educativas como parte de su formación pedagógica.

Nivel de formación predoctoral. Incluye a aquellos docentes y directivos que han transitado por los diferentes niveles del sistema propuesto. Los resultados en este nivel de formación pedagógica son: ii) Profundizar en los fundamentos teóricos y metodológicos que sustentan el proceso de investigación en educación, desde los diferentes paradigmas que lo soportan y la utilización práctica de métodos y técnicas científicas y ii) Desarrollar habilidades de búsqueda, selección y evaluación de la información para la construcción de un marco teórico referencial de la investigación durante el proceso de formación doctoral y la producción y comunicación de artículos científicos.

\section{EVIDENCIAS DEL DESARROLLO DEL SISTEMA}

Desde el inicio de la ejecución del sistema para la formación del claustro se realizó un ciclo de conferencias, seminarios, talleres, cursos y se profundizó en el trabajo didáctico de las carreras. En el mismo participaron profesores de las carreras y directivos académicos de la universidad, en función de mejorar el nivel pedagógico del claustro y de la transformación de los indicadores que permiten el acceso y la retención del estudiantado en las cohortes de la Universidad de Otavalo. En la figura 2 se ofrece una ilustración del proceso realizados en la puesta en práctica del sistema de formación pedagógica diseñado para el claustro de profesores de la Universidad de Otavalo, que sirve de referencia para el análisis de los 
indicadores que se tuvieron en cuenta en el seguimiento y la evaluación del mismo, revelados en el modo de actuación profesional de los docentes.

ENFOQUE TRADICIONAL DE LA FORMACIÓN PEDAGÓGICA EN LA UNIVERSIDAD DE OTAVALO

CARACTERIZACIÓN INICIAL DE LAS COMPONENCIAS DOCENTES DE LOS PROFESORES EN FORMACIÓN
DE LA UNIVERSIDAD DE OTAVALO

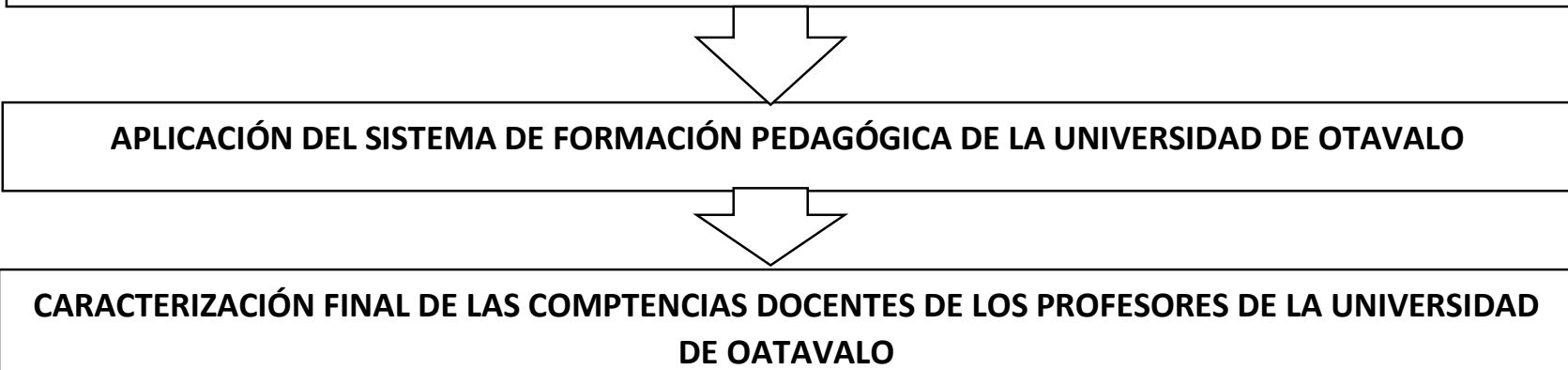

Fig. 2: Proceso de aplicación del sistema de formación pedagógica.

Entre las principales estrategias y acciones desarrolladas por parte del equipo de asesores se encuentran las siguientes: (1) Asesoramiento didáctico a directores de carreras; (2) Actividades metodológicas sobre los lineamientos de la evaluación del proceso de evaluación de los aprendizajes en los estudiantes; (3) Talleres para la confección de materiales de estudio para la preparación de los estudiantes en competencias generales. (Guías de Estudio, Base Orientadora para la Acción. BOA); (4) Preparación didáctica del equipo de docentes para enfrentar el proceso de capacitación a estudiantes de todas las carreras en competencias generales; (5) Preparación didáctica del equipo de docentes encarados de la confección, aplicación y revisión de examen de competencias generales; (6) Revisión de las líneas de investigación de la Universidad y un taller metodológico sobre la vinculación docencia investigación con la comunidad; (7) Curso de tutores, asesores y lectores (oponentes) de tesis. El producto final fue la revisión y reelaboración de guía metodológica para la realización de trabajos de titulación en la Universidad de Otavalo; (8) Asesoramiento metodológico para la preparación de la planificación docente en la Universidad; (9) Curso sobre marco lógico para la gestión de proyectos; (10) Talleres de revisión y análisis crítico de las mallas curriculares de todas las carreras. Presentación del informe final; (11) Confección de propuesta de reglamento de evaluación del desempeño docente; (12) Confección de modelo por competencias para la Universidad; (13) Curso taller de modelo por competencias para la elaboración de los planes de estudio de las carreras. Confección de los planes de estudio de todas las carreras; (14) Asesoría para diseñar programas analíticos (Sílabos) de las asignaturas. Completamiento de los programas analíticos (Sílabos) de las asignaturas a impartir; (15) Definición de procedimiento para realización de examen de conocimientos relevantes y traslados de carrera; y (16) Revisión y perfeccionamiento del proyecto del curso de nivelación para la admisión de estudiantes en la Universidad de Otavalo.

\section{CONTROL Y SEGUIMIENTO}

La dirección del proceso de formación de los profesores universitario requiere conocer cómo se realiza el aprendizaje de los docentes que participan en los cursos propuestos y cuáles son sus resultados. Para ello la evaluación del aprendizaje es imprescindible. Esta es una parte esencial del proceso de enseñanza que permite el control y seguimiento de la asimilación de los conocimientos, de habilidades y hábitos que se adquieren como resultado del proceso y el grado en que se logran los objetivos propuestos. Constituye un elemento de retroalimentación y dirección del proceso mediante el cual se conoció: i) A los profesores, como destinatarios y sujetos activos de su aprendizaje, sus necesidades, intereses, expectativas y su experiencia anterior, incluyendo sus aciertos y errores; ii) Al proceso de aprendizaje que comprende los propósitos, los medios y los elementos que favorecen o dificultan dicho aprendizaje; y iii) Los resultados del proceso de formación pedagógica entendidos como cambios relevantes y significativos en la experiencia y en las capacidades de los profesores. Permitió además: i) Valorar los elementos que constituyen la actividad educativa, desde su planificación y programación en los distintos niveles, hasta los resultados, pasando por la aplicación y puesta en práctica de las estrategias y actividades previstas. En esta valoración se destacaron los criterios cualitativos y la determinación de las necesidades de mejora; y ii) Decidir acerca de las medidas de ayuda individualizada necesarias para lograr el desarrollo óptimo en cada profesor, en 
función de sus posibilidades. Este ámbito también comprende las decisiones, los cambios en la programación y en la práctica docente, en la metodología y en los recursos, así como en las calificaciones, promoción y titulación correspondientes.

El control y seguimiento se realizó sistemáticamente, se mantuvo un monitoreo continuo por los niveles de dirección de la institución y se emplearon diferentes modalidades como la rendición de cuenta al HCUS (Honorable Consejo Universitario Superior) mensualmente por la Vicerrectoría Académica, las visitas a clases sin previa comunicación, las entrevistas formales e informales a estudiantes y profesores, la aplicación de encuestas dirigidas a conocer el grado de satisfacción de profesores y estudiantes en relación con la implementación del proceso de formación pedagógica (profesores) y respecto a la calidad de las clases (estudiantes). El sistema se puso en práctica teniendo en cuenta los indicadores que establecen los órganos de control de la Educación Superior en el Ecuador. Se ajustaron con frecuencia las estrategias seguidas y se logró una propuesta flexible que garantizó la retroalimentación y la adecuación inmediata a las necesidades y demandas del entorno.

\section{RESULTADOS}

Los resultados se sustentaron en la aplicación de métodos científicos y técnicas de investigación como: el método de criterios de expertos (Método Delphi) por la posibilidad que ofrece de obtener información de manera independiente, de intercambio de la misma y de evitar evaluaciones superficiales y la Técnica de ladov para conocer el grado de satisfacción de los profesores que participaron en el proceso de formación pedagógica. Se realizó una medición de los cursos, conferencias, seminarios y talleres planificados por niveles de formación después de haber recibidos los contenidos de los cursos impartidos, que permitió contrastar el grado de satisfacción de los profesores.

El Método Delphi permitió extraer la información de los expertos que conforman un grupo heterogéneo, analizar las convergencias de opiniones en torno a la propuesta que se aborda en la investigación, facilitó a los expertos entrevistados emitir sus opiniones sin conocer lo que otros colegas planteaban y favoreció un consenso de ideas, reflexiones, criterios que influyó en la solución de la problemática planteada. Se basó en la utilización sistemática e iterativa de juicios de opiniones de un grupo de expertos hasta llegar a un acuerdo. Se trató de evitar las influencias de individuos o grupos dominantes y existió la retroalimentación hasta llegar al acuerdo final. Los pasos lógicos seguidos para la aplicación del Método Delphi fueron: la concepción inicial del sistema propuesto, la caracterización de la preparación del claustro de profesores con énfasis en el campo de acción contextualizado, la descripción de las vías que lo conforman y la fundamentación pedagógica teniendo incluyendo los fundamentos filosóficos, psicopedagógicos y didácticos en que se sustenta el sistema.

Los expertos solicitaron el anonimato, pero se creyó oportuno informar su procedencia y las características distintivas. De los 12 expertos, 5 son de cubanos, poseen el grado científico de Doctores en Ciencias y una experiencia de más de 20 años en la Educación Superior, 3 son ecuatorianos, Doctores en Ciencias y su experiencia docente es de más de 20 años en la Educación Superior, 3 son españoles, Doctores en Ciencias, con una experiencia de más de 20 años en la Educación Superior y 1 es de Estados Unidos que posee el grado científico de Doctores en Ciencias y una experiencia profesional de más de 20 años en la Educación Superior.

Los 7 criterios emitidos por los expertos después de realizar las rondas correspondientes, atendiendo a la metodología establecida, se ponderaron y validaron. Los expertos consideran:

Criterio 1: Que el Sistema que se propone es muy pertinente, novedoso y aplicable en el contexto ecuatoriano.

Criterio 2: Que en el diagnóstico realizado debió ampliarse el muestreo a todas las Universidades posicionadas legalmente en este territorio.

Criterio 3: Que debe incluirse en un anexo lo relacionado con el Reglamento de Admisión y Nivelación para el acceso de los estudiantes a la Universidad de Otavalo.

Criterio 4: Que se deben mencionar las formas de control, seguimiento y evaluación empleadas en la implementación del Sistema.

Criterio 5: Que el Sistema propuesto puede ser aplicado ya que es muy pertinente, importante y novedoso para todas las Universidades del Ecuador.

Criterio 6: Que el Sistema posee potencialidades para lograr la transformación de la dimensión pedagógica del claustro de profesores de la Universidad de Otavalo. 
Criterio 7: Los 9 expertos extranjeros (Cuba (5), España (3) y EEUU (1)) que participan en el proceso establecieron comparaciones con sus países resaltando la percepción, concepción y originalidad en las vías utilizadas para la implementación del Sistema.

Los resultados obtenidos al aplicar el paquete estadístico orientado al ámbito de las Ciencias Sociales SPSS para Windows versión 15.0, evidenciaron que el comportamiento del coeficiente de variación, valor de las ligaduras y el coeficiente Kendall o coeficiente de correlación de rango y demostraron el alto grado de concordancia de los expertos con los criterios emitidos.

Se aplicaron otros métodos de investigación científica como de los niveles teóricos y empíricos como:

Métodos del nivel teórico: El método histórico-lógico para determinar la evolución del proceso de formación pedagógica en la Universidad de Otavalo y la didáctica relacionada con su aplicación anterior al momento de la realización de la nueva propuesta. El sistémico para diseñar el nuevo sistema de formación pedagógica, en el que todos sus componentes tienen relación armónica. La modelación para representar el sistema de formación pedagógica. El analítico-sintético para la interpretación de la información suministrada por los métodos y técnicas que sirvieron para el diagnóstico relacionado con la aplicación del sistema de formación pedagógico en la Universidad de Otavalo y el inductivo-deductivo para determinar las potencialidades y las limitaciones del proceso de formación pedagógica en la universidad de Otavalo y proponer sobre esa base el nuevo sistema de formación pedagógica.

Se observaron clases aplicando una guía con indicadores establecidos como parte del contenido del sistema formación pedagógica, para evaluar el ascenso de la calidad de la docencia durante la puesta en práctica del sistema. Se realizó el análisis documental para el estudio de documentos relacionados con los antecedentes de la formación pedagógica en la universidad de Otavalo y con el contenido del nuevo sistema. Se aplicaron encuestas a estudiantes, profesores y directivos para recoger información relacionada con el impacto trasformador del nuevo sistema de formación pedagógica en las actividades sustanciales de la Universidad de Otavalo y la triangulación de fuentes de información para garantizar la confiabilidad de los resultados en la evaluación de las competencias docentes de los profesores vinculados la ejecución del sistema de formación pedagógica.

En la evaluación de los resultados de la aplicación del sistema de formación pedagógica se consideraron los siguientes indicadores, en el modo de actuación de los docentes bajo la influencia del mismo, se comprobó el ascenso a niveles superiores de la demostración profesional cotidiana y un desempeño superior en los siguientes aspectos.

(1) Demostración del desarrollo de los componentes de la competencia cognoscitiva en el tratamiento de la información científica del contenido de la materia que imparte cada profesor. Desempeño en la localización, selección e interpretación crítica de la información existente en diferentes fuentes escritas, orales, audiovisuales, gráficas, virtuales. Procesamiento y registro de la información científica en forma de esquema didáctico, resumen, párrafo o cita textual y elaboración de la información científica sobre el contenido de su materia y su presentación en materiales docentes y medios de enseñanza.

(2) Demostración del desarrollo de los componentes de la competencia para el diseño del proceso pedagógico, para concebir el contenido de la materia que imparte en la formación de los estudiantes. Desempeño en la selección del contenido y la formulación de los objetivos en el estudio del contenido de la materia que imparte, teniendo en cuenta el diagnóstico integral y contextualizado, en la elaboración de tareas docentes que precisen el método, los procedimientos, las fuentes y las formas para el aprendizaje de los estudiantes y en la determinación de indicadores y escalas para el convenio de evaluación con los alumnos en el estudio de un tema.

(3) Demostración del desarrollo de los componentes de la competencia comunicativo-orientadora para conducir el proceso pedagógico diseñado, para el estudio del contenido de la materia que imparte. Desempeño para orientar, motivar, conducir y controlar el aprendizaje activo y desarrollador del contenido, en los contextos del grupo escolar, la escuela, la familia y la comunidad, en la creación de condiciones para el desarrollo de relaciones en un clima favorable sustentado en el respeto, en la comprensión, en la confianza y en la autoridad profesional para el estudio y como modelo de comunicador en su expresión oral, escrita y corporal en la conducción del estudio.

(4) Demostración de los componentes de la competencia para la interacción social en el tratamiento del contenido de la materia que imparte. Desempeño en la orientación y motivación de los alumnos mediante el estudio del contenido de su materia, hacia un objetivo de carácter colectivo priorizado por la sociedad, en relación con el uso del mismo en función del perfeccionamiento de la organización y el funcionamiento de los procesos sustantivos de la Universidad y en el desarrollo de actos académicos, debates y reflexiones mediante la incorporación dinámica del nuevo conocimiento. 
(5) Desarrollo de los componentes de la competencia investigativa en el tratamiento del contenido de la materia que imparte. Desempeño en la planificación de la investigación del proceso pedagógico relacionado con el estudio del contenido de su materia, en el procesamiento científico de la información obtenida en la investigación del proceso pedagógico relacionado con el estudio de su materia y en la comunicación e introducción de los resultados investigativos en la transformación del proceso pedagógico en el contenido de la materia bajo su responsabilidad.

\section{CONCLUSIONES}

En este artículo se expresan los fundamentos teóricos metodológicos que sustentan la formación docente con carácter pedagógico, mediante el análisis y contextualización de concepciones teóricas científicas para sustentar el sistema de cursos, conferencias, seminarios y talleres dirigidos a elevar la de preparación del claustro de profesores de la Universidad de Otavalo, Se realizó un diagnóstico que determinó las regularidades con respecto a los niveles de formación de los profesores que conforman el claustro y permitió establecer el sistema formación para transformar la realidad profesional de los profesores. Se fundamentaron los cursos, conferencias, seminarios y talleres dirigidos a mejorar el modo de actuación pedagógico de los docentes del centro. Se validó el mismo aplicando métodos de investigación cualitativos y cuantitativos, así como técnicas que garantizaron los resultados del proceso. Se muestran resultados que validan la pertinencia y el impacto que ha tenido en la formación del claustro de la institución, la implementación del sistema propuesto que influyó en los resultados satisfactorios de la Universidad de Otavalo en la recién finalizada evaluación institucional, realizada por los organismos de revisión y control de la educación superior en Ecuador.

\section{REFERENCIAS}

Abreu, O. La dinámica sociedad, universidad, enseñanza-aprendizaje de la historia. www.uotavalo.edu.ec, Revista Sarance, ISSN0252-8630, (en línea), 31, 101-112, junio de (2014).

Addine, F y otros 9 autores. Didáctica Teoría y Práctica., 1ra Edición, Pueblo y Educación, 1-291, La Habana, Cuba (2004).

Álvarez, C. La Pedagogía como ciencia. Epistemología de la Educación, 1ra Edición, Pueblo y Educación, 1-254, La Habana, Cuba (1999).

Álvarez, R. Metodología de la enseñanza y el aprendizaje, 2da Edición, Universidad Simón I. Patiño, 1-128 Cochabamba, Bolivia (2014).

Alvariñas, M y Fernández, M. A. Percepciones de Futuros Maestros ante Nuevos Contextos de Aprendizaje, DOI: 10.4067/S0718-50062011000400006, Revista Formación Universitaria, (en línea), 4(4), 37-46 (2011).

Arenas, M. V. y Fernández, T. "Formación Pedagógica Docente y Desempeño Académico de Alumnos en la Facultad de Ciencias Administrativas de la UABC", http://www.redalyc.org, Revista de la Educación Superior, ISSN 0185-2760, (en línea), 38 (2), 150, 7-18, abril-junio (2009).

Chehaybar, E y Amador, R. Procesos y prácticas de la formación universitaria, Revista Pensamiento Universitario, ISBN 970-32-32-0372-8, (en línea), 3 (93), 147-177, México (2003).

García, L. Autoperfeccionamiento docente y creatividad, 1ra Edición, Pueblo y Educación, 1- 85, La Habana, Cuba (1996).

Lucchesi, M. A. S. La Universidad Internacional en América Latina: un Nuevo Paradigma para el Siglo XXI, DOI: 10.4067/S0718-50062011000100005, Revista Formación Universitaria, (en línea), 4 (1), 25-36 (2011).

Moreno, C., Molina, Y. y Chacón, J. Impacto del estilo pedagógico integrador en los estudiantes de licenciatura en Educación Básica de la Facultad de Estudios a Distancia, DOI: 10.4067/S071850062014000600005, Revista Formación Universitaria, (en línea), 7 (6), 37-44 (2014).

Pedraja, L. M., Araneda, C. A., Rodríguez, E. R. y Rodríguez, J.J. Calidad en la Formación Inicial Docente: Evidencia Empírica en las Universidades Chilenas, DOI: 10.4067/S0718-50062012000400003, Revista Formación Universitaria, (en línea), 5 (4), 15-26 (2012).

Pla, R. y otros 16 autores. Una concepción de la Pedagogía como ciencia desde un enfoque histórico cultural. 1ra Edición, Pueblo y Educación, 1-79, La Habana, Cuba (2010).

Ramos, J., Abreu, O. y Dorta, M. Proyecto Educativo Integral para la Planificación Estratégica, Evaluación y Acreditación Universitaria, ponencia, Congreso Internacional Pedagogía 2013, 1-12, La Habana, Cuba, 3 al 10 de febrero (2013).

Rosenshine, B., Meister, C., \& Chapman, S. Teaching students to generate questions: A review of the intervention studies. Review of Educational Research, 66(2), 181-221 (1996). 